\title{
La deuda pública en Ecuador: ¿Se cumplió la regla fiscal?
}

\section{Public debt in Ecuador: Was the fiscal rule fulfilled?}

\author{
Gonzalo Jonás Paredes Reyes ${ }^{12 *}$ y Karolyne Nikole Saltos Sánchez ${ }^{1}$ \\ ${ }^{1}$ Universidad de Guayaquil \\ ${ }^{2}$ Universidad Católica de Santiago de Guayaquil \\ *gonzalo.paredes01@cu.ucsg.edu.ec
}

DOI: https://doi.org/10.26871/killkana_social.v2i3.342

\begin{abstract}
Resumen
El objetivo de este trabajo es analizar el cumplimiento de la normativa vigente con respecto al "techo" del endeudamiento público en el Ecuador. La pregunta de investigación es: ¿las autoridades económicas inobservaron lo establecido en el código de finanzas públicas para determinar el monto de la deuda pública, y su límite en el período de 2010 al 2017 ? La sostenibilidad del endeudamiento está interrelacionada con la política fiscal, y al manejo de la cuenta corriente, lo que exige una revisión de la gestión de las finanzas públicas estatales desde lo conceptual, hasta los alcances de su metodología. La importancia de esta investigación radica en precisar uno de los indicadores más relevantes para la evaluación de la sostenibilidad macroeconómica, y la capacidad de pago de un país. Para llevar a cabo esta investigación se realizó un análisis comparativo entre la ley de finanzas públicas del 2010, y el marco legal vigente anterior a esta última. Además, se revisó cada uno de los cuerpos legales vinculados a la estimación del monto de la deuda pública, así como las diferentes visiones y/o enfoques sobre esta cuantificación. Por lo tanto, se desarrolló un enfoque de investigación cualitativo, que implica una revisión documental. Se concluyó que las autoridades económicas respetaron el límite legal del endeudamiento público al situarse por debajo del umbral del $40 \%$, la legislación secundaria hizo cumplir lo que dispone el código de finanzas públicas y la Constitución de la República y, la cuantificación estuvo de acuerdo a lo que recomienda el manual del Fondo Monetario Internacional (FMI).
\end{abstract}

Palabras clave: deuda pública, coeficiente de deuda, Ecuador.

\begin{abstract}
The objective of this paper is to analyze compliance with current regulations regarding the calculation of Ecuador's public debt. The research question is: did the economic authorities ignore the provisions of the public finance code to determine the amount of public debt, and its limit in the period from 2010 to 2017? The sustainability of indebtedness is interrelated to the sustainability of the fiscal policy plans, and to the management of the current account, which requires a review of the management of state public finances from the conceptual point of view, to the scope of its methodology. The importance of this research lies in specifying one of the most relevant indicators for the evaluation of macroeconomic sustainability, and the ability to pay a country. To carry out this research, a comparative analysis was made between the public finance law of 2010 and the legal framework in force prior to the latter. In addition, each of the legal bodies linked to the estimation of the amount of public debt was reviewed, as well as the different views and / or approaches to this quantification. Therefore, a qualitative research approach was developed, involving a documentary review. It was concluded that the economic authorities respected the legal limit of public indebtedness to be below the threshold of $40 \%$, the secondary legislation enforced the provisions of the public finance code and the Constitution of the Republic and, the quantification was in accordance with what is recommended in the manual of the International Monetary Fund (IMF).
\end{abstract}

Key words: public debt, debt ratio, Ecuador.

\section{Introducción}

A partir de 2000, el Fondo Monetario Internacional (FMI) exigió a los países en desarrollo implementar la constricción de la política fiscal. Estos requerimientos se derivaron de la experiencia argentina en los noventa y su crisis: la culpa del explosivo endeudamiento público fue del excesivo gasto público. Esto sin verificar la hipótesis de la "deuda eterna e ilegítima". Por lo tanto, el FMI asesoró a las autoridades económicas del Ecuador (dolarizado) en
2001 para asegurar el pago de la deuda externa a través de severas reglas macrofiscales: el límite al crecimiento del gasto público y el "techo" del $40 \%$ de deuda pública sobre el PIB. También se pidió al Ecuador que corrigiera la presentación y análisis de las estadísticas de las finanzas públicas.

En la práctica, hasta el 19 de octubre de 2016, para el cumplimiento del límite del endeudamiento, el cálculo del monto de la deuda pública se tomaba de los totales de 
la externa y de la interna. Sin embargo, el FMI en un informe para el Ecuador en 2002 recomendó que "siguiendo las normas internacionales, las estadísticas de las finanzas públicas son una consolidación y no una agregación de las transacciones de las instituciones de los respectivos subsectores del SPNF". Con el Código de Planificación y Finanzas Públicas (COPLAFIP) y su reglamentó se retomó lo que siempre el FMI recomendó en sus manuales y guías de compilación.

¿Por qué las autoridades ecuatorianas no implementaron lo que el FMI recomendaba y lo que el reglamento de la Ley Orgánica de Responsabilidad, Estabilización y Transparencia Fiscal estipulaba? ¿Por qué es necesario consolidar? ¿El manual del FMI identifica a la consolidación como una ciencia "perfecta"? Por otro lado, si la consolidación fue y es recomendada por qué se insiste en agregar, en vez de consolidar. Desde el COPLAFIP, la pregunta central de la investigación es ¿se cumplió con la regla fiscal del $40 \%$ ?

Para determinar si se cumplió o no, se llevará a cabo una investigación documental: revisión y análisis de manuales, guías e informes, y misivas entre autoridades de las instituciones públicas. Es importante resaltar que se realizó entrevistas a dos ministros de economía de la última década. De esto se desprende lo inédito de este estudio, se lo volcó a la academia dado que la política ha dominado el análisis técnico de las autoridades gubernamentales.

La importancia de este estudio es contribuir al avance del estudio de las finanzas públicas y a la mejora en la presentación de las estadísticas del sector público: el análisis de sostenibilidad debe partir de montos consolidados de la deuda pública, y no de agregados. Se concluye que el reglamento, y su posterior reforma mediante decreto, hacen cumplir la ley (COPLAFIP). No la contradice. Por lo tanto, el Gobierno Nacional del periodo 2007-2017 cumplió con el límite establecido en dicho cuerpo legal y aceptó las recomendaciones del FMI para la mejora de las estadísticas de las finanzas públicas.

El trabajo está estructurado en dos partes: los antecedentes describen el marco normativo, y la estructura institucional desde el año 2002, presentando a la LOREYTF como la norma previa que presenta las normas macrofiscales señaladas en el COPLAFIP, en la segunda parte los resultados presentan dos apartados, el primero concierne al Código de Finanzas y la norma reglamentada de la consolidación, le sigue una revisión de la metodología de la consolidación desarrollada en los Manuales de Estadística de las Finanzas Públicas (MEFP) y Guías de Compilación del FMI, y finalmente una contextualización de la dinámica de la consolidación normada por el COPLAFIP y su normativa secundaria, y las recomendaciones del Fondo Monetario Internacional.

\section{La primera versión del cálculo del monto de la deuda pública y el "techo" del $40 \%$}

A inicios del siglo XXI con la adopción del nuevo esquema monetario (dolarización total y unilateral), el Mi- nisterio de Finanzas y Crédito Público pasó a denominarse Ministerio de Economía y Finanzas en enero del 2002, creándose subsecretarías de Inversión Pública y de Política Económica. Con este cambio inicia la visión planificadora del gobierno con respecto a la programación fiscal, y de inversión de obras públicas como funciones de la Cartera de Estado. La Ley Orgánica de Responsabilidad, Estabilización y Transparencia Fiscal (LOREYTF) expedida en el 2002 establece algunas reglas macrofiscales y crea el Fondo de Estabilización, Inversión Social y Productiva y Reducción del Endeudamiento Público (FEIREP), cuyos ingresos provenían de $45 \%$ del exceso de ingresos petroleros sobre los presupuestados y del superávit presupuestario derivados del petróleo trasportado por el Oleoducto de Crudos Pesados (OCP), cuyo parámetro de distribución según su art. 17, era destinar el $70 \%$ de los recursos para la recompra de la deuda pública externa e interna, el $20 \%$ para emergencias del país, y $10 \%$ para la inversión en salud y educación.

Estos cambios representaron un nuevo direccionamiento de los usos del financiamiento público, para (2015), "esta ley fue la primera reforma en debilitar la supremacía sobre el capital" (p. 260), los procesos legislativos anteriores en dolarización han sido dominados por una tendencia hacia la austeridad fiscal trabajando prioritariamente para cubrir el servicio de la deuda. La institucionalización del uso de los nuevos recursos petroleros pretendía disminuir el monto del endeudamiento público cumpliendo con los compromisos pactados de recompra de deuda externa hasta alcanzar una reducción equivalente al $40 \%$ del PIB (Ley Orgánica de Responsabilidad, Estabilización y Transparencia Fiscal (LOREYTF), 2002, Art. 7).

Esto además significó una revalorización de los bonos de deuda ecuatorianos, generando utilidad sobre los tenedores de deuda, pues existía una garantía a nivel de ley orgánica de que los haberes se iban a cubrir por medio de ingresos recurrentes. Los recursos del FEIREP no eran presupuestados en la proforma del Gobierno central, sino que eran gestionados bajo un fideicomiso administrado por el Banco Central, sumado a las preasignaciones de esta ley, representaba el ejercicio de una política fiscal anticíclicas. En el art. 4 de LOREYTF, el crecimiento del gasto primario estaba limitado al 3,5\% del crecimiento anual real considerando además la inflación, lo que significaba una escasa posibilidad para la inversión social.

Años más tarde, en 2005 el FEIREP es remplazado por CEREPS, la Cuenta de Reactivación Productiva y Social, ley reformada y enviada como ley económica urgente aprobada por el Congreso Nacional, en donde de forma esencial, se ingresan los recursos petroleros al Presupuesto General del Estado en una cuenta independiente de la Cuenta Corriente Única del Tesoro Nacional eliminándose el fideicomiso, la nueva distribución destina el $35 \%$ para la cancelación de deuda con el IESS, recompra de deuda pública interna y externa, y financiamiento de proyectos de inversión; el $30 \%$ para inversión social en donde el $15 \%$ corresponde a educación, y el otro $15 \%$ a la salud; 
$20 \%$ para fondos de estabilización de emergencias; $5 \%$ para investigación en ciencia y tecnología, $5 \%$ para mejoramiento vial; y otro $5 \%$ para reparación ambiental por efecto de actividades hidrocarburíferas, aquellos rubros que no fueren utilizados para sus fines alimentarían el fondo de estabilización (Ley Orgánica Reformatoria a la Ley Orgánica de Responsabilidad, Estabilización y Transparencia Fiscal, 2005).

Uno de los elementos en el manejo del endeudamiento, es lo que comprendía deuda pública, en su art. 5 la define como la deuda externa e interna que incluye la deuda con el IESS y todas aquellas obligaciones asumidas por el Estado, conforme a la ley, a excepción de los pasivos de la Agencia General de Depósitos (AGD). Sobre estos rubros, la preasignación estatal de reducción de endeudamiento público tendría efecto, el Ministerio de Economía debía disminuir como mínimo en un $16 \%$ el saldo de la deuda pública total hasta el término del año 2006, y en los siguientes periodos de gobierno debería plantearse el mismo fin hasta que la deuda pública llegue al $40 \%$ del PIB.

Pese a que el objetivo de la ley era reducir el endeudamiento con las preasignaciones destinadas al pago de la deuda, el rubro sí se disminuye en casi 17 puntos porcentuales del 2003 hasta el año 2006 como se muestra en el Gráfico 1, sin embargo pese a que la deuda externa en el 2000 siendo \$11228,8 millones disminuyó al 2005 en $\$ 10850$ millones, la deuda interna incrementó \$854 millones en el mismo periodo, aumento principalmente originados en Certificados de Tesorería (Cetes), que tienen vencimientos de hasta un año plazo, el monto de estos fue de \$19 millones en el 2000, alcanzando \$680 millones a fines del 2005. A pesar de la existencia de los fondos petroleros para recompra de deuda interna utilizados en este periodo por un valor de $\$ 700$ millones, la deuda pública total creció de $\$ 14174$ millones a $\$ 14536$ millones; atribuyéndose la disminución del umbral del $40 \%$ de la deuda pública/PIB al crecimiento nominal de la producción nacional.

Figura 1. Evolución de la Deuda Pública Total del Ecuador 1995-2010

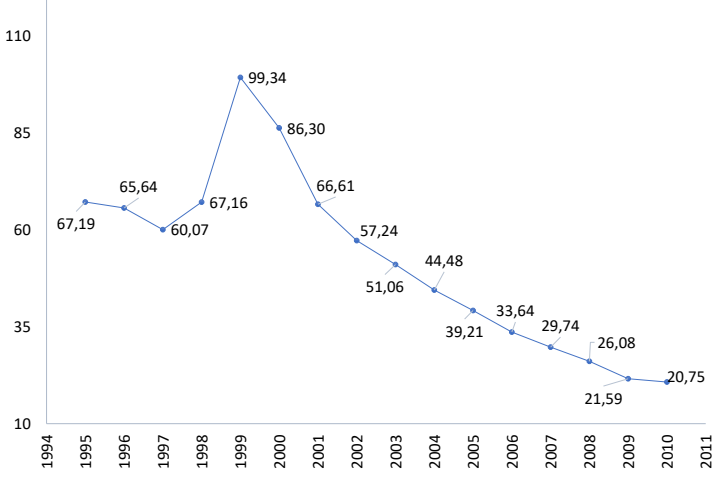

Fuente: Ministerio de Finanzas, Boletín Estadístico al 2010
De esta manera podemos observar, que no necesariamente una mayor utilización de los ingresos para el pago del endeudamiento disminuye la deuda pública, los límites en esta ley estaban cubiertos para los ingresos y gastos presupuestarios más no para el pago de la deuda, la regla para el crecimiento de la deuda pública fue estrictamente una sugerencia del Fondo Monetario Internacional, que bajo las condiciones para la firma de un acuerdo de financiamiento, nunca generado, y las sugerencias en el marco de sus Informes anuales de Perspectivas Económicas Mundiales del año 2002 y 2003, concluyen que una proporción de la deuda externa por encima del $40 \%$, la probabilidad condicional de llegar a una crisis de deuda se eleva a alrededor de 15-20 por ciento (2002), es uno de los pocos registros en donde se concluye este umbral, pero que ha sido tomado, y escasamente estudiado para las reglas macro fiscales de los programas económicos mundiales.

En el año 2007, se elabora la proforma presupuestaria del año 2008 con el objetivo de financiar la creación de empleo, la producción y el mayor acceso a servicios sociales por medio de una distribución sostenible y más equitativa de los recursos. En noviembre de ese año, se firma un convenio de participación interinstitucional junto al Contralor General del Estado Carlos Pólit, para normar la ejecución del Sistema Integrado de Gestión Financiera (SIGEF). A inicios del 2008, el Ministerio tecnifica la gestión presupuestaria y transparenta la gestión de las finanzas públicas, por medio del manejo de la Cuenta Corriente Única del Tesoro, y de la implementación del nuevo Sistema de Administración Financiera del Sector Público con la aplicación del sistema informático eSIGEF y eSIPREN. La Asamblea Constituyente por medio de una ley orgánica incorpora al Presupuesto General del Estado (PGE) los fondos petroleros como ingresos de capital a fin de priorizar, e intensificar la inversión pública, creándose el Comité de Deuda y Financiamiento. Por Decreto Ejecutivo de enero del 2008, el Ministerio de Economía y Finanzas pasa a denominarse Ministerio de Finanzas.

Noviembre del 2008 se profundiza la gestión pública de la deuda por medio de un informe de la deuda comercial preparado por la Comisión para la auditoría integral de Crédito Público (CAIC) que sirvió como un elemento que refuerza la decisión de la en ese entonces, ministra de finanzas María Elsa Viteri, anunciando que el gobierno se acogería a un plazo de 30 días de mora técnica para el pago del servicio de los bonos Global 2012 y 2030, para junio del 2009 presenta a la Presidencia el informe sobre la estrategia de solución de la deuda comercial, y sus resultados obtenidos, en donde Ecuador retira el $91 \%$ de la deuda en bonos.

\section{Resultados}

\subsection{La consolidación de la deuda en el COPLAFIP}

Como antesala del COPLAFIP está la Ley Orgánica para la Recuperación del Uso Público de los Recursos Petroleros del Estado, aprobada en el 2008. Un proyecto de ley 
que buscaba agilizar la expansión del gasto público, hacia los sectores más prioritarios. Buscaba que la planificación de la política económica permitiera la apertura de mayor inversión pública, evidenciándose en la nueva regla del crecimiento del gasto estipulada en el Código del 2010, donde permiten que los gastos sean financiados por los ingresos según su propia naturaleza.

En mayo de ese año, se posesiona Patricio Rivera como ministro de finanzas, el MEF y la dirección General del IESS firman el acta de compromiso de pago relacionado a los valores del $40 \%$ de contribución estatal a las pensiones jubilares. Para octubre, entra en vigencia el Código Orgánico de Planificación y Finanzas Públicas (COPLAFIP), el cuál deroga las leyes creadas hasta ese entonces, e integra la planificación institucional con la gestión presupuestaria plurianual.

El (Código Orgánico de Planificación y Finanzas Públicas [COPLAFIP], 2010) presenta tres reglas macrofiscales esenciales para el manejo de las finanzas públicas, las que corresponden al límite para el monto del saldo total de la deuda pública del conjunto de organismos del sector público hasta un $40 \%$ del PIB (art. 123), en el 2014 se establece el reglamento a la ley, el cual establece nociones conceptuales acerca de lo que es deuda pública y aquello que no lo es. En el año 2016, el presidente de la República reforma ciertos elementos del reglamento, habilitado constitucionalmente por sus competencias, y establece la medición del endeudamiento por medio del Manual de Estadísticas y Finanzas Públicas del FMI (MEFP), el cual establece el uso de la deuda consolidada como instrumento de medición de la contabilidad gubernamental por medio del (Decreto Ejecutivo Nro. 1218, 2016).

Desde mayo del 2018 la publicación del examen a la deuda pública de la Contraloría General del Estado (CGE) establecía indicios de que la ley no fue cumplida, y que la regla macrofiscal del $40 \%$ fue irrespetada por medio del Decreto 1218, el informe asevera que las discrepancias metodológicas respecto al cálculo del endeudamiento se deben a que tanto el Código de Finanzas, como su Reglamento General modifican, eliminan y/o crean disposiciones normativas presentes en su marco legal anterior hasta la LOREYTF.(2018) detalla el siguiente comentario:

La LOREYTF, en su art. 5 definía que para determinar el límite del endeudamiento se debía considerar la deuda externa e interna, incluyendo la del IESS, el reglamento posteriormente reformado bajo el decreto ejecutivo, reforma su art. 135 estableciendo un método de cálculo de la deuda pública no prevista en la ley.

Resulta confusa la comparación que se realiza en el Informe, ambas leyes tanto el COPLAFIP y la LOREYTF tienen una naturaleza de orden orgánico, no es la primera vez que se crea una ley sin derogar las anteriores a ella, el COPLAFIP al ser expedida, en su disposición derogatoria, deroga las leyes de igual o menor jerarquía entre ellas están: Ley Orgánica de Administración Financiera y Control (LOADFC), Ley Orgánica de Responsabilidad, Estabilización y Transparencia Fiscal (LOREYTF), Ley Orgánica para la Recuperación del Uso de los Recursos Petroleros del Estado y Racionalización Administrativa de los Procesos de Endeudamiento, Ley de Presupuestos del Sector Público, y el capítulo I de la Ley de Regulación Económica y Control del Gasto Público.

Con respecto al Decreto Ejecutivo 1218 (2016), hace dos reformas esenciales, la primera en donde monto total del saldo de la deuda pública del conjunto de entidades y organismos del sector público no sobrepase el $40 \%$ del producto interno bruto (PIB), se añade que "el cálculo respectivo se lo efectuará sobre la base de los Estados Consolidados de Deuda Pública correspondiente a cada ejercicio fiscal" (art. 4), y lo segundo es que se deberá considerar el Manual de Estadísticas de las Finanzas Pública del Fondo Monetario Internacional (art. 4).

El MEFP edición 1986, introduce el término de consolidación, y aunque tiene un alcance esencialmente conceptual "el mecanismo y el significado de la consolidación son iguales" a su edición posterior del 2001 (2008). Y es el mismo (2002a) que en su visita a Ecuador de observancia y evaluación del manejo de los datos y estadísticos que manejan las instituciones del Ministerio de Finanzas y Economía (MEF), INEC, y Banco Central del Ecuador (BCE), durante el período comprendido entre el 11 y el 25 de abril de 2002 , publica un informe recomendando como de alta prioridad:

- Compilar y divulgar de manera regular estadísticas para los diferentes niveles de gobierno (incluyendo gobierno central consolidado y gobierno general) con suficiente grado de detalle y siguiendo la sectorización, clasificación y presentación recomendados en el MEFP 1986, o de preferencia MEFP 2001.

- Elaborar y divulgar estadísticas sobre financiamiento y deuda (externa e interna; corto, mediano y largo plazo) del gobierno central por tipo de tenedor de deuda y por tipo de instrumento según lo recomienda el MEFP 1986, o de preferencia MEFP 2001 (p. 24).

Surge entonces la pregunta del por qué estas recomendaciones no se aplicaron, ni se consideraron, si en las observaciones del documento del Departamento de Estadística (2002) indicaba que la compilación de las estadísticas de las finanzas públicas en términos generales siguen conceptos y definiciones nacionales similares a los que contiene el MEFP 1986, pero que no existe una actualización hacia la nueva edición del manual, en donde además señala que "siguiendo las normas internacionales, las estadísticas de las finanzas públicas son una consolidación y no una agregación de las transacciones de las instituciones de los respectivos subsectores del $\operatorname{SPNF}^{1}$ "(p. 16), lo cual en sus recomendaciones claves establece que debe programarse una ruta de aplicación del Manual de Estadísticas de las Finanzas Públicas del 2001

\footnotetext{
${ }^{1}$ Sector Público No Financiero
} 
Estas observaciones fueron publicadas días previos a la introducción a la LOREYTF, la preocupación del FMI era que las reformas aumentaran el riesgo de duplicación en cuanto al registro y compilación de las estadísticas públicas, y que el nuevo estatuto del Ministerio de Finanzas en obligación a la compilación de las cuentas nacionales podría duplicar funciones con el BCE, lo mismo ocurría con la compilación de las estadísticas de la deuda externa, la cual manejaban ambas (Informe sobre la Observancia de los Códigos y Normas (IOCN)-Módulo de Datos, ROSC por sus siglas en inglés, 2002).

Esta práctica de la consolidación, sí fue considerada en la presentación de la (Ley Orgánica de Responsabilidad, Estabilización y Transparencia Fiscal (LOREYTF), 2002) donde en su séptima disposición transitoria el MEF hasta 31 de octubre del 2002 debía elaborar el estado consolidado de la deuda pública en los términos que establece el artículo 5 , con el fin de cumplir con las metas anuales y cuatrianuales de reducción de la deuda pública; en su (Reglamento a la Ley Orgánica de Responsabilidad, Estabilización y Transparencia Fiscal [R. LOREYTF], 2003) expedido por Decreto Ejecutivo Nro. 96 el art. 12 disponía:

Los estados consolidados de la deuda pública serán elaborados y publicados por la unidad correspondiente del Ministerio de Economía y Finanzas en coordinación con el Banco Central del Ecuador, hasta 60 días después de finalizado cada mes, mismos que servirán de base para calcular la relación deuda/PIB según lo dispuesto en el art. 5 de la ley (p. 25).

La consolidación no es un término reciente, y pese a que existía esta base no hay registros actuales de que esta práctica se haya ejecutado, la normativa de aquel entonces respaldaba el uso de los estados consolidados para el cálculo del índice Deuda/PIB tanto en la ley como en su reglamento, las presunciones de que el Código del COPLAFIP tergiversaba una práctica prudente de las finanzas es darle la razón a la LOREYTF, cuyas disposiciones en la gestión y los límites del endeudamiento hoy son coherentes con la normativa vigente que incluye el Decreto Nro. 1218.

El Código Orgánico de Planificación y Finanzas Públicas [COPLAFIP] (2010) también tenía elementos relacionados a la consolidación, en el art. 74 las enumeraciones de las funciones del Sistema Nacional de Finanzas Públicas (SINFIP) rectorado por el Ministro de finanzas, establecía que entre sus deberes estaba la preparación de las estadísticas fiscales, consolidación de la información presupuestable, contable, financiera y de la deuda pública de todas las entidades sujetas al código, y la elaboración de los Estados Financieros Consolidados de los organismos del Sector Público No Financiero (SPNF). Y que, según el art. 157, la agregación y consolidación de los estados financieros son obtenidos en relación a las agrupaciones predefinidas y los requerimientos de la administración del Estado, es decir del Gobierno Central, dirigido por la función ejecutiva quién tiene la competencia de reglamentar las leyes aprobadas por el Congreso Nacional, así en art. 147 de la (Constitución del Ecuador, 2008).

El Reglamento General al COPLAFIP expedido en el 2014 , en su texto original ${ }^{2}$ presenta disposiciones relacionadas a la consolidación, en la proforma presupuestaria sólo los balances debidamente auditados y definitivos formarán parte del monto consolidado concerniente a los convenios de deuda, aquellos que no cumplan con los requisitos y deba realizarse el pago, se lo efectuará como un anticipo para luego ser registrado y su anticipo amortizado inmediatamente (art. 131), la consolidación no pretende eliminar las obligaciones de pago, es un punto de orden para gestionar de forma ordenada y sistemática el servicio de las obligaciones financieras que tiene el Estado.

En el espíritu de la reforma que hace el decreto al art. 133 sigue siendo el Ministerio de Finanzas quien elabora los estados agregados y consolidados de la deuda, el énfasis a la consideración de que sean los estados consolidados los que sirvan de base para el cálculo del índice relación deuda/PIB por medio de las consideraciones del Manual de Estadísticas de las Finanzas Públicas del FMI, es un formalización de la práctica que venía y/o suponía aplicarse desde 1986, muestra de ello es el art. 158 del Reglamento al Código Orgánico de Planificación y Finanzas Públicas (2014), este apartado describe la consolidación en donde el Ministerio de Finanzas en cumplimiento de sus deberes de consolidar la información del sector público, en la presentación de dicho informe debe . eliminar todas las transacciones y relaciones otorgante-receptor que tienen lugar entre las entidades objeto de la consolidación"(p. 37 ), existe desde ya una naturaleza de evitar el riesgo de duplicación de registro de las operaciones que existen en el Sector Público no Financiero (SPNF), y el Sector Público Financiero (SPF), es decir una consolidación intersectorial, el FMI amplía esta clasificación, la cuál será revisada junto a la consolidación intrasectorial brevemente explicada en el artículo citado en el siguiente apartado.

\subsection{La consolidación de la deuda pública en el MEFP}

Las estadísticas de las finanzas públicas contribuyen a detectar fuentes tempranas de vulnerabilidad, y al desarrollo de acciones que mejoren las condiciones sobre las cuales son calculadas. El Departamento de Estadística del FMI busca ampliar la disponibilidad de datos confiables, trabajando en manuales que describan la metodología para la compilación de estadísticas tanto económicas como financieras sobre actividades del sector público y del gobierno general, desarrollando: Manual de Estadística de Finanzas Públicas (MEFP), el Manual de Estadísticas Monetarias y Financieras (MEMF), Manual de Balanza de Pagos y Posición de Inversión Internacional (MBPPII), el Manual de Cuentas Nacionales Trimestrales (MCNT), y las Estadísticas de la deuda del sector público: Guía para compiladores y usuarios que se armonizan con el Sistema de Cuentas Nacionales (SCN) según su vigencia y edición,

\footnotetext{
${ }^{2}$ No reformado por el Decreto Ejecutivo 1218.
} 
la actual es la del 2008. El MEFP busca "dar mayor claridad a las normas que rigen la compilación y presentación de las estadísticas fiscales"(p. 21) reforzando las iniciativas de trasparentar y mejorar la información financiera del sector público, así el (International Monetary Fund, 2014) ïnstó a los países miembros a adoptar las directrices de este manual como base para compilar y divulgar estadísticas de las finanzas públicas, así como para declarar esta información al FMI" (p. 21), pues es adoptado en la presentación de sus datos fiscales, norma aprobada por el Directorio Ejecutivo del FMI.

Son tres ediciones del Manual de Estadísticas de Finanzas Públicas, siendo la primera la de 1986, la segunda del 2001, y la última publicada en el 2014; que actualmente son concebidas como una serie de directrices internacionales emitidas por el FMI para la construcción de un marco estadístico macroeconómico especializado, el marco de las Estadísticas de Finanzas Públicas (EFP). El Manual se complementa con otros recursos como Guías de Compilación, asistencia técnica y labores de capacitación individuales, centrándose en ofrecer descripciones conceptuales utilizadas en la compilación y divulgación de las EFP (2014).

Los datos registrados en el sistema de EFP se determinan como flujos y/o saldos, existen reglas contables que son utilizadas para determinar la valoración, el registro y otros aspectos relevantes. Entre las reglas contables destacan el momento de registro de los flujos, para Ecuador los ingresos y gastos se registran sobre la base devengada ${ }^{3}$, esta unificación de información cuenta con el Clasificador Presupuestario y el Catálogo único de Cuentas, registrándose según su naturaleza y grupo en el sistema de eSIGEF actualizando sus modificaciones de forma actualizada.

La regla contable del (International Monetary Fund and International Monetary Fund, 2003) presentaba a la agregación, es decir valores agregados como medida para resumir los valores de los flujos que han sido registrados en el Sistema EFP, mientras la consolidación está enmarcada como una práctica de presentación de datos método que sirve para "presentar las estadísticas de un conjunto de unidades como si formaran una sola unidad" (p.47) y "elimina la doble contabilización dado que un flujo o saldo de una unidad se empareja con el flujo o saldo correspondiente registrado por la segunda unidad con que está siendo consolidado, y ambos flujos y/o posiciones de saldos se eliminan" (International Monetary Fund, 2014). Ecuador desde el 2002 participa y observa las recomendaciones de las Normas Especiales para la Divulgación de Datos (NEDD) del FMI producto de su visita, en donde aclara que en la elaboración de las EFP de las unidades que conforman los subsectores del SPNF "se netean las transferencias entregadas y las transferencias recibidas entre las distintas unidades que conforman el respectivo subsector.

${ }^{3}$ Para la contabilidad gubernamental sólo serán registrados los hechos económicos en el momento que ocurran, haya o no un movimiento de dinero consecuente a las condiciones con los que fueron pactados.
Así pues, las EFP representan una consolidación y no una agregación”, así recomendado en el MEFP 1986 (2003, p.95).

El MEFP recomienda la presentación consolidada de las estadísticas del sector gobierno general y a cada uno de sus subsectores, las unidades del sector público que son incluidas se presentarán de forma separada, es decir las sociedades públicas financieras y sociedades públicas no financieras; de forma conjunta los que conformen el gobierno general como parte del sector público consolidado, las estadísticas se presentarán consolidadas dentro de cada grupo. La compilación de las estadísticas del sector público y/o gobierno general pueden presentar dos clases de consolidación: la intrasectorial y la intersectorial, en la cual la intrasectorial es la primera en realizarse entre las unidades que conforman un subgrupo, y la intersectorial entre aquellos subgrupos que conforman un sector, sea este el gobierno general o sector público (International Monetary Fund, 2014).

En el Manual de Contabilidad Gubernamental del Ecuador del (2006), los principios de Contabilidad Gubernamental aplicados al sector gubernamental nacional son las bases que rigen la materia contable, entre ellas se encuentra la consolidación, la cual es preparada según las necesidades de la administración en función de los requerimientos específicos, sea para obtener información financiera consolidada para diversos niveles de agregación de datos, a nivel institucional sectorial y global "con agregados netos de las cuentas patrimoniales, de los resultados de gestión, así como de los informes presupuestarios producidos por los entes financieros objeto de consolidación" (2016, p.86), conforme a los siguientes ámbitos:
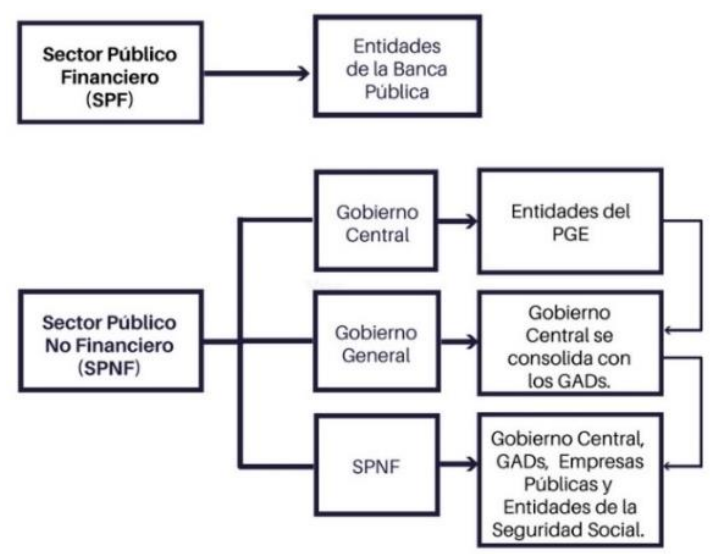

Figura 2. Consolidación de la Información Financiera del Sector Público

Adaptado de: : Ministerio de Finanzas, 2016.

Estos datos son validados por medio de un análisis de sus registros contables, pasando a su vez por la agregación comprendida como la acumulación de valores de cada grupo, subgrupo y cuentas contables del estado financiero generando los grupos institucionales establecidos en la consolidación, y la eliminación de los flujos financieros de los 
Estados de Resultados y Flujo del Efectivo de las transacciones entre las entidades del Sector Público con el fin de reflejar resultados netos; generándose así cuatro informes consolidados, el del SPF, SPNF, Gobierno General y del Gobierno Central (Ministerio de Finanzas, 2016). El proceso de consolidación guarda armonía con el MEFP 2014 donde es mejor eliminar los movimientos internos (dentro del sector público), incluyendo sólo aquellos valores de origen del sector externo, o privado, es decir, los que cruzan la frontera con sectores no residentes.

La consolidación tiene como objetivo no sólo eliminar la doble contabilización de los flujos al interior de un grupo institucional, busca ser práctico, pues "los recursos dedicados a la consolidación y el nivel de detalle aplicado a esta deben ser proporcionales a su importancia fiscal"(International Monetary Fund, 2014), esta afirmación deja la puerta abierta a la interpretación, en donde la consolidación desde el MEFP cuenta con un acercamiento conceptual, parcialmente metodológico donde no se diferencia lo que el sistema nacional admite consolidar, y aquello que no. En sí mismo, la consolidación no afecta a las partidas de resultado, pues estas partidas resultantes de la agregación deben ser las mismas obtenidas por consolidación, buscando que la unidad analítica resultante refleje útilmente el impacto global de las operaciones gubernamentales sobre la economía (Estadística de las Finanzas Públicas: Guía de Compilación para países en desarrollo, 2011).

La regla macrofiscal del límite del endeudamiento en el COPLAFIP señala que:.$^{\text {el }}$ monto total del saldo de la deuda pública del conjunto de entidades y organismos del sector público, no sobrepase el cuarenta por ciento (40\%) del PIB"(art. 124), en la consolidación de las estadísticas de la deuda, que es el elemento que se añade en la reforma a su reglamento, deben de presentarse de acuerdo de cada cobertura institucional (International Monetary Fund y International Monetary Fund, 2013), en donde la sectorización del sector público para efectos de la consolidación comprende al SPNF, y al gobierno general que contiene el gobierno central, las dependencias estatales y los gobiernos locales (Wickens, 2008), guardando relación con la Figura 1 en la consolidación que hace el Ministerio de Finanzas.

Llama la atención que en la introducción al capítulo de consolidación de la deuda pública en la Guía de Compilación del FMI conforme al MEFP, el FMI (2013) señala "La consolidación es una ciencia imperfecta" que data desde la primera edición del Manual, lo que sugiere que debe de servirse de otros recursos y herramientas estadísticas, conceptuales, prácticas y legales para hacerla más eficiente y útil para la administración estatal, y de sus recursos.

Este proceso, según normas internacionales, se destaca como la última etapa dentro del proceso de compilación, resaltando nuevamente que debe tenerse sentido práctico: "los recursos destinados y el grado de detalle aplicado a la consolidación deben ser directamente proporcionales a su importancia numérica"(International Monetary Fund y International Monetary Fund, 2013). Una vez terminada la agregación horizontal (suma de los valores de las distintas unidades del sector público) de las estadísticas de la deuda se inicia el proceso de la consolidación, obteniendo así las estadísticas consolidadas, las cuales serían iguales si no existieran saldos o flujos recíprocos entre las unidades cuyas estadísticas se compilan, pero que es sumamente improbable.

Los elementos que deben consolidarse, constituyen pasivos que son instrumentos de deuda, los cuales pueden convertirse en activos financieros para la contraparte, el acreedor. Son descritos en probable orden de importancia, y para ambos tipos de consolidación (intrasectorial e intersectorial) según (International Monetary Fund y International Monetary Fund, 2013) en la siguiente figura 3:

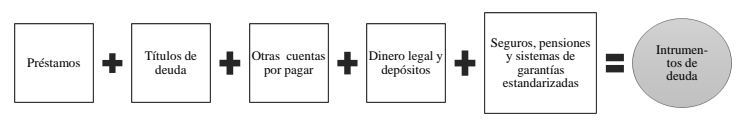

Figura 3. Pasivos instrumento de deuda consolidables

Fuente: Estadística de la Deuda Pública del Sector Pública. FMI, 2013

El objetivo no es necesariamente lograr una consolidación perfecta, sino identificar aquello que es significativo para los agregados finales, los Derechos Especiales de Deuda pese a ser reconocidos por el FMI como instrumentos de deuda, no se consolidan pues su acreedor suele ser el resto del mundo. Al consolidar, es posible efectuar la consolidación intersectorial e intrasectorial, estas combinaciones se ilustran en la Tabla 1 derivada de (International Monetary Fund y International Monetary Fund, 2013), los saldos o flujos resultantes de actividades de endeudamiento cuyo acreedores son sociedades públicas financieras, en "principio son eliminados" (p. 162).

Tabla 1 Consolidación intrasectorial e intersectorial de las estadísticas de la deuda del sector público

\begin{tabular}{lcc}
\hline \multicolumn{1}{c}{ Unidades del sector público } & $\begin{array}{c}\text { Consolidación } \\
\text { intrasectorial }\end{array}$ & $\begin{array}{c}\text { Consolidación } \\
\text { intersectorial }\end{array}$ \\
\hline Sector Gobierno general & $\mathrm{x}$ & $\mathrm{x}$ \\
Gobierno Central & $\mathrm{x}$ & \\
Gobierno central presupuestario & $\mathrm{x}$ & \\
Gobierno central extrapresupuestario & $\mathrm{x}$ & \\
Fondo de seguridad Social & $\mathrm{x}$ & \\
Gobierno estatal & $\mathrm{x}$ & \\
Gobierno local & $\mathrm{x}$ & \\
Sociedades públicas & $\mathrm{x}$ & $\mathrm{x}$ \\
Sociedades públicas no financieras & & $\mathrm{x}$ \\
Sector público no financiero & $\mathrm{x}$ & \\
Sociedades públicas financieras & & \\
Sector público & & \\
\hline
\end{tabular}

Fuente: Estadística de la Deuda Pública del Sector Público. FMI, 2013

\subsection{Revisando los métodos}

Para complementar el uso de la consolidación en la presentación de las estadísticas de la deuda pública, y 
que estas sirvan de base para el cálculo del monto de la deuda pública, y su límite del $40 \%$ Deuda pública/PIB, revisaremos las unidades que bajo el criterio de la (Informe Final del examen especial a legalidad, fuentes y usos de la deuda pública interna y externa, durante el periodo 1 de enero de 2012 y 24 de mayo de 2017, 2018) son deuda pública y que la figura de consolidación inobserva estos rubros de endeudamiento por medio de la consolidación, así ilustrados en la tabla 2, en donde la deuda bruta sería el concepto de deuda pública aplicable a la ley:

Tabla 2 Visión de la Composición de la Deuda Pública

\begin{tabular}{lccc}
\hline \multicolumn{1}{c}{ Elementos } & $\begin{array}{c}\text { Decreto } \\
\text { (Consoli- } \\
\text { dada) }\end{array}$ & $\begin{array}{c}\text { FMI } \\
\text { (Cuasia- } \\
\text { gregada) }\end{array}$ & $\begin{array}{c}\text { CGE } \\
\text { (Bruta) }\end{array}$ \\
\hline Preventas petroleras & No & Sí & Sí \\
IESS & No & Sí & Sí \\
Banca Pública & No & Sí & Sí \\
CETES & No & Sí & Sí \\
Pasivos Contingentes & No & No & Sí \\
Atrasos Internos (Deuda Flotante) & No & Sí & Sí \\
\hline
\end{tabular}

Adaptado de: Decreto 1218, 2016; MEFP FMI 2018, Informe de CGE 2018

El World Economic Outlook Database (2018) publicó cifras de deuda ecuatoriana en su página web, estableciendo que "la definición de deuda del FMI incluye preventas de petróleo, certificados del tesoro, préstamos del Banco Central y atrasos internos que están excluidos de la definición oficial de deuda". Esto sumado a los Informes de Contraloría pretende señalar el incumplimiento a los dispuesto por el COPLAFIP.

\subsection{Preventas Petroleras}

El gobierno del Ecuador acordó una operación comercial y de endeudamiento con el Banco Internacional de China, en donde se operacionalizó ventas anticipadas de petróleo, bajo el art. 130 del texto original del Reglamento al Código Orgánico de Planificación y Finanzas Públicas (2014) "las ventas anticipada de un bien o servicio de una empresa pública no se considera deuda pública", por tanto eran razón suficiente para no consolidarse como deuda pública, Así también, el art. 137 del Código Orgánico de Planificación y Finanzas Públicas [COPLAFIP] (2010) permitía concretar estas operaciones de convenio de crédito vinculándolos con otros convenios o contratos comerciales de exportación, y otros los cuales no son deuda pública, siendo el Ministerio de Finanzas el ente rector que expidiera los procedimientos que normen esas contrataciones.

\subsection{Obligaciones con el IESS}

La deuda que el Ecuador mantiene con el IESS constituía un componente de la deuda pública en la LOREYTF del 2002 a miras de cancelarse de forma progresiva por una deuda heredada del cambio a la dolarización, esta deuda fue eliminada de los balances del IESS en 2016 tras conclusiones de las entidades supervisoras, para junio del 2017 se determina que los valores liquidados no estaban extintos, y su contabilización en los balances debía restituirse. Estos montos están dentro de los $\$ 8000$ millones que el Estado debe cancelar al IESS, así anunciando por el Ministerio de Finanzas en mayo del 2018, este monto lo componen dos rubros esenciales, el primero es por el aporte fijo de del $40 \%$ al ente por concepto de pensiones jubilares, y el segundo corresponde a préstamos al Banco del Instituto Ecuatoriano de Seguridad Social (BIESS) por inversiones en títulos del Estado, y empréstitos que constituirían pasivos del Estado.

La clave es identificar si esto corresponde a valores de deuda pública, pues para la asignación del $40 \%$, una parte del debate lo identifica como mero dinero de los afiliados y jubilados y por tanto no sería aplicable la consolidación al carecer de la característica primordial de ser rubros de una entidad interna del sector público. Los que están a favor de ser eliminada su contabilización en la deuda pública, señalan que siendo parte del sector público, el ente funciona de forma autónoma, y el aporte fijo del $40 \%$ para el fondo de pensiones cuenta como una garantía del Estado en donde entregaría recursos cuando el IESS no fuera capaz de asignarlos, el IESS otorga esta prestación de forma total (Ley de Seguridad Social, 2001), es decir, en sí mismo no es una deuda contratada, para el Estado este rubro podría ser un pasivo contingente por su naturaleza, COPLAFIP en su art. 124 excluye los pasivos contingentes de la contabilización.

Los pasivos contingentes sujetos a garantías de prestaciones sociales pueden influir en el funcionamiento de la economía en general, pero no se registran en las estadísticas de las finanzas públicas mientras no se produzca el acontecimiento específico que lo condiciona, representan riesgos fiscales y son el resultado de políticas públicas deliberadas (International Monetary Fund, 2014). Para el MEFP 2014, las contribuciones sociales por fondos de pensión del gobierno, o seguridad social "nunca se consolidan"(p. 70), pues redireccionan esta figura, tratándola como si fueran pagos al empleado en el sector hogares, y luego entregados por el empleado al sistema de seguridad social.

En el caso de la deuda al BIESS, la seguridad social no forma parte de las entidades presupuestarias según el art. 77 del Código Orgánico de Planificación y Finanzas Públicas [COPLAFIP] (2010), pues sus recursos son autónomos y distintos al fisco amparado en el art. 161, al igual que la extensión de una institución especializada como el BIESS y los pasivos contratados con el Banco Central del Ecuador (BCE), pero en su sectorización estos sí pertenecen al sector público del Estado así señalado por el (Evaluaciones detalladas utilizando el Marco de Evaluación de la Calidad de los Datos, 2003), donde a pesar de que este último es un banco, no se dedica a la intermediación financiera sino a la realización de préstamos al sector público local, cuyas recursos son transferencias e ingresos provenientes del Estado. El FMI, respecto a operaciones que sirvan para cubrir obligaciones en casos de incumplimiento de 
pagos pactados con gobiernos, sean locales, estatales o del banco central, son consideradas como pasivos contingentes implícitos.

Existen otros elementos al alcance del análisis, sin embargo, los citados nos brindan algunas pautas para determinar si otros componentes son incluidos o eliminados en la consolidación de la deuda pública, en la Guía de Compilación de las estadísticas de la Deuda del Sector Público del FMI (2013) destinan un capítulo a la consolidación de la deuda, pero su contenido no refleja de una forma más clara y contextual acerca de cómo debe consolidarse. El Decreto que reforma el Reglamento al COPLAFIP menciona que será aplicable sobre el "monto total del saldo de deuda pública del conjunto de entidades y organismos del sector público", este texto original está sujeto a ser interpretado en distintas formas, pues el saldo podría implicar una operación de consolidación en donde se netean los valores, así como el de una agregación en valores brutos; el conjunto de las entidades también podría implicar una sectorización sujeta a la normativa y a razones explicadas de una clasificación contemplada en la consolidación.

Para María Elsa Viteri, exministra de economía la conclusión del cumplimiento de la regla del $40 \%$ es un tema de corte legal, y las acciones tomadas son determinadas en el marco legal en el que nos encontramos. Es necesario depurar el plan de cuentas sistematizado en el e-SIGEF, pues por ejemplo las preventas petroleras tienen un orden diferido, la clasificación entonces debe determinar si se clasifican en pasivos diferidos, como una operación comercial; al igual que revisar el resto de los pasivos del Estado. El problema para ella, radica en que no se define, y la validez de las normas están sujetas a interpretaciones, por tales razones consultó tanto a las autoridades del FMI como a la CGE acerca de las formas de aplicación de la normativa en debate, cuestionamiento que en términos técnicos específicos no fueron respondidos (Viteri, 2018).

\section{Conclusiones y recomendaciones}

La consolidación es un método contable de presentación de las Estadísticas de las Finanzas Públicas (EFP), establecidas y sugeridas para su aplicación en los países miembros del FMI. Los actuales manuales, y guías de compilación tienen un alcance conceptual y parcialmente metodológico, que sirve como pauta y norma internacional del tratamiento integral de los saldos y flujos económicos, pues los agregados macroeconómicos finales del Sector Público No financiero (SPNF) constituyen una consolidación más no una agregación de sus operaciones.

La consolidación busca eliminar la doble contabilización de los flujos y/o saldos al interior y entre instituciones gubernamentales para reflejar eficientemente el desempeño del Estado frente a las instituciones fuera de ella, el sector privado y del exterior. Los recursos destinados a este proceso de consolidación o no consolidación deben ser transparentados con dos razones fundamentales: la de su relevancia e importancia fiscal, y que la unidad analítica resultante refleje el impacto global de estas operaciones gubernamentales.

La consolidación es una herramienta sujeta a las interpretaciones, y necesidades de la administración gubernamental de cada país y de cada periodo, el FMI aún no ha desarrollado un modelo estándar para la presentación de los datos de deuda consolidados, la herramienta al alcance está en la consolidación de los sectores que pertenecen al gobierno general y al Sector Público No Financiero como complemento a la compilación técnica de las estadísticas fiscales, por tanto es necesaria una revisión fundamentada en los objetivos y fines del Estado desarrollado en un marco legal claro y específico, y en el estudio integral de los manuales y normas internacionales aceptados para la práctica.

La regla macrofiscal del límite a la deuda pública fue cumplida según la normativa vigente, la reforma efectuada al reglamento por el decreto fue jurídicamente viable, amparado constitucionalmente el ejecutivo tiene plena competencia de reglamentar el código orgánico del COPLAFIP, cuya norma según la jerarquización normativa debe guardar compatibilidad con los convenios internacionales sujetos al Artículo IV del Convenio Constitutivo del FMI. Sin embargo, es necesario afinar los conceptos y disposiciones normativas presentes en el marco jurídico en el que nos encontramos, estableciendo no sólo definiciones, también las cláusulas que ameritan el cumplimiento de dichas normas.

\section{Referencias Bibliográficas}

Assessing Sustainability. (2002, may). Publicación de Policy Development and Review Department, 2002.

Código Orgánico de Planificación y Finanzas Públicas [COPLAFIP]. (2010, oct).

Decreto Ejecutivo Nro. 1218. (2016, oct).

Estadística de las Finanzas Públicas: Guía de Compilación para países en desarrollo. (2011). Washington.

Evaluaciones detalladas utilizando el Marco de Evaluación de la Calidad de los Datos. (2003, mar). Ecuador.

Informe Final del examen especial a legalidad, fuentes y usos de la deuda pública interna y externa, durante el periodo 1 de enero de 2012 y 24 de mayo de 2017. (2018, apr). Quito.

Informe sobre la Observancia de los Códigos y Normas (IOCN)-Módulo de Datos, ROSC por sus siglas en inglés. (2002, mar). Quito.

International Monetary Fund (Ed.). (2014). Government finance statistics manual 2014. Washington D.C: International Monetary Fund.

International Monetary Fund, y International Monetary Fund. (2013). Public Sector Debt Statistics: Guide for Compilers and Users. Washington, D.C.: International Monetary Fund. (OCLC: 985998367)

International Monetary Fund and International Monetary Fund. (2003). Manual de Estadísticas de Finanzas Públicas, 2001. Washington, D.C.: International Monetary Fund. (OCLC: 951626271) 
Ley de Seguridad Social. (2001, nov).

Ley Orgánica de Responsabilidad, Estabilización y Transparencia Fiscal (LOREYTF) (Vol. Ley N² 2002-83). (2002, jun).

Ley Orgánica Reformatoria a la Ley Orgánica de Responsabilidad, Estabilización y Transparencia Fiscal. (2005, jul).

Manual de Contabilidad Gubernamental. (2006, oct).

Normativa de Contabilidad Gubernamental (Vol. Acuerdo Ministerial Nro. 0067). (2016, apr).

Paredes, G. J. (2015). Integraciones monetarias pasivas en Sudamérica (1991-2011) (1era edición ed.). Buenos Aires: C.A.B.A., Argentina: Ediciones Cooperativas, abril.

Reglamento a la Ley Orgánica de Responsabilidad, Estabilización y Transparencia Fiscal [R. LOREYTF] (Vol. Decreto Ejecutivo Nro. 69). (2003).

Reglamento al Código Orgánico de Planificación y Finanzas Públicas. (2014, nov).

Wickens, T. (2008, oct). Manual de Estadísticas de las Finanzas Públicas 2001. Material Suplementario,
Consolidación de las Estadísticas del Sector Público No Financiero.

World Economic Outlook Database. (2018). Report for Selected Countries and Subjects.

Recibido: 30 de junio de 2018

Aceptado: 3 de septiembre de 2018 